\title{
Performance Comparison Between Hama and Hadoop
}

\author{
Shuo Li and Baomin $\mathrm{Xu}$ \\ School of Computer and Information Technology, Beijing Jiaotong University \\ Beijing 100044 \\ 2889130209@qq.com
}

\begin{abstract}
Massive scientific computations such as matrix, graph and network algorithms are very attractive when they come to modelling real-world data. Apache Hama is a pure BSP (Bulk Synchronous Parallel) distributed computing framework for massive scientific computations. In this paper, our experiments were conducted on a 4-node Hadoop cluster. We implement Monte Carlo algorithm of Pi in Hama and Hadoop under the same software and hardware environment. The experimental results show that Hama can achieve much higher performance than Hadoop in our testbed.
\end{abstract}

Keywords: Hama; Hadoop; BSP; matrix computation; performance.

\section{Introduction}

The growing demand for large-scale data processing has led to create a new parallel computing model MapReduce [7] for data intensive computing. MapReduce is a successful paradigm to perform large-scale data processing. However, MapReduce model does not efficiently support iterative computations [11]. Large scale scientific computations such as most machine learning and data mining applications involve iterative computations are often used as primary means for many data-intensive scientific applications. The computation core of many data-intensive scientific applications can be expressed as matrix/graph computations based on iterative aggregation-disaggregation methods. Matrix/graph computation has been a focus area in the HPC (High-performance computing) community for many years. With the prevalence of MapReduce, a cloudbased parallel computing framework [1,15], the studies on the high-performance massive scientific computations using cloud have been conducted $[2,3,8,9,10,13]$.

Hama [2] is a distributed framework on Hadoop [4], the open source implementation of MapReduce, for high-dimensional matrix and graph computations. Like Pregel [3], Hama is heavily based on BSP model and also has some similarity to Hadoop. The key difference between Hadoop and Hama is BSP tasks can communicate to each other while MapReduce tasks cannot communicate with each other. Hama aims at a powerful tool for various scientific applications and providing the BSP primitives for developers, so messaging between tasks and a synchronization barrier. Owing to the Hama is a BSP [5] implementation over Hadoop, the BSP nodes and jobs management, including fault tolerance, and node-to-node communication directly leverage the facilities offered by Hadoop. The synchronization barrier is enforced using Zookeeper[6]. The architecture of Hama is illustrated in Fig.1. Hama supports MapReduce engine, BSP engine, and Dryad[12] engine. The MapReduce engine is used for matrix computations while BSP and Dryad engines are used for graph computations.

The performance of distributed computing frameworks impacts many important applications. In this paper, we choose matrix computations as the target for evaluation the performance of Hama. To evaluate the efficiency and accuracy of iterative algorithm on Hama, the selected algorithm needs to have a fixed execution results and suitable for parallelization. So, we select the Monte Carlo calculation of Pi [14] as experimental 
algorithm. Specifically, we share our experience of implementing Monte Carlo Calculation of Pi with Hama and Hadoop, and present our preliminary results.

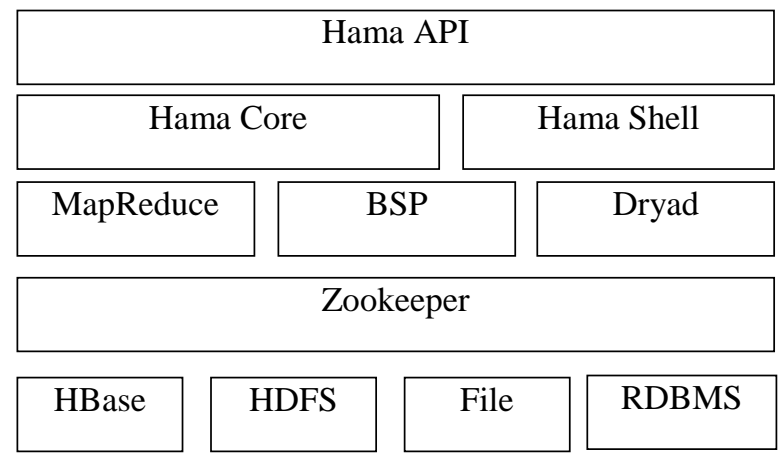

Figure 1. The Architecture of Hama

The remaining of this paper is organized as follows: Section 2 gives a detailed description of the Monte Carlo Calculation of Pi. Section 3 describes the experimental results. Section 4 draws the conclusions.

\section{Monte Carlo Calculation of Pi}

The Monte Carlo method provides an approximate solution to a variety of mathematical problems by performing statistical sampling experiments on a computer. The calculation of Pi using the Monte Carlo method can be described as:

If a circle of radius $r$ is inscribed inside a square with side length $2 r$, then the area of the circle will be $\pi r^{2}$ and the area of the square will be $(2 r)^{2}$.The ratio of the area of the circle to the area of the square is $\pi / 4$. This means that, if you randomly pick points $\left\{\left(x_{i}, y_{i}\right)\right\}_{i=1}^{n}$ inside the square. With approximately $\pi / 4$, those points should fall inside the circle, because the circle has $\pi / 4$ the area of the square. So, we can estimate $\pi$ as:

$$
\pi \approx 4 \frac{\mathrm{m}}{\mathrm{n}}
$$

Where $\mathrm{n}$ is the number of points inside the square. $\mathrm{m}$ is the number of points that satisfy $x_{i}^{2}+y_{i}^{2} \leq 1$ in circle.

To calculate each significant digit there will have to be about 10 times as the number of iterations to calculate the preceding significant digit.

\section{Experimental Results}

The evaluation of Hama has been performed on 4-nodes Hadoop Cluster. Each node consists of a quad core processor Intel Xeon X3470 and 16GB of main memory. The operation system is Redhat Enterprise Linux 5. Hadoop-0.20.2 and Hama-0.4.0incubating have been installed on the cluster. In addition, the number of both Hadoop's map and Hama's BSPPeer are fixed at 20.

In this evaluation, we used both Hama and Hadoop to implement Monte Carlo calculation of Pi. Through many experiments, we found that the time growth trends can be divided into three stages. Correspondingly, the number of iterations is divided into three intervals: 1) advantage interval $0 \sim 10^{5}$. Hama gives better performance than Hadoop; 2) available interval $10^{5} \sim 1.5^{*} 10^{7}$. The performance of Hama is gradually reduced as the problem size becomes larger; 3) disadvantage interval $1.5^{*} 10^{7} \sim 10^{8}$. The performance of Hadoop is better than that of Hama. 


\subsection{Advantage Interval}

The experimental results of both Hama and Hadoop algorithm are shown in Table 1 and Table 2, respectively, and graphed in Fig.2.

Table 1. Advantage Interval - Hama

\begin{tabular}{|c|c|c|c|c|c|c|c|}
\hline number of iterations & \multicolumn{6}{|l|}{ Hama } & \multirow{2}{*}{$\begin{array}{l}\text { mean value } \\
3.3652\end{array}$} \\
\hline & elapsed time (seconds) & 3.381 & 3.352 & 3.363 & 3.369 & 3.361 & \\
\hline 0 & value of $\mathrm{Pi}$ & N/A & N/A & N/A & N/A & N/A & N/A \\
\hline & elapsed time (seconds) & 3.364 & 3.368 & 3.358 & 3.359 & 3.367 & 3.3632 \\
\hline 10 & value of $\mathrm{Pi}$ & 3.2600 & 3.3400 & 3.2200 & 3.2600 & 3.0200 & 3.2398 \\
\hline & elapsed time (seconds) & 12.202 & 12.171 & 12.175 & 12.184 & 12.171 & 3.3612 \\
\hline $10^{2}$ & value of $\mathrm{Pi}$ & 3.17 & 3.188 & 3.13 & 3.132 & 3.186 & 3.1612 \\
\hline & elapsed time (seconds) & 12.181 & 12.178 & 12.178 & 12.179 & 12.172 & 3.3664 \\
\hline $10^{3}$ & value of $\mathrm{Pi}$ & 3.1389 & 3.1419 & 3.1461 & 3.1449 & 3.1457 & 3.1435 \\
\hline & elapsed time (seconds) & 12.180 & 12.182 & 12.183 & 12.170 & 12.171 & 3.364 \\
\hline $10^{4}$ & value of $\mathrm{Pi}$ & 3.1441 & 3.1387 & 3.1444 & 3.1403 & 3.1422 & 3.1413 \\
\hline & elapsed time (seconds) & 12.180 & 12.178 & 12.174 & 12.177 & 12.168 & 3.3578 \\
\hline $10^{5}$ & value of $\mathrm{Pi}$ & 3.1423 & 3.1404 & 3.1417 & 3.1429 & 3.1413 & 3.1417 \\
\hline
\end{tabular}

Table 2. Advantage Interval - Hadoop

\begin{tabular}{|c|c|c|c|c|c|c|c|}
\hline number of iterations & \multicolumn{6}{|l|}{ Hadoop } & \multirow{2}{*}{\begin{tabular}{|l} 
mean value \\
64.4734 \\
\end{tabular}} \\
\hline & elapsed time (seconds) & 64.275 & 64.776 & 64.368 & 64.705 & 64.243 & \\
\hline 0 & value of $\mathrm{Pi}$ & N/A & N/A & N/A & N/A & N/A & N/A \\
\hline & elapsed time (seconds) & 64.26 & 64.233 & 64.265 & 64.173 & 64.254 & 64.237 \\
\hline 10 & value of $\mathrm{Pi}$ & 3.27 & 3.35 & 3.58 & 3.24 & 3.17 & 3.322 \\
\hline & elapsed time (seconds) & 64.103 & 64.259 & 64.233 & 64.285 & 64.216 & 64.2192 \\
\hline $10^{2}$ & value of $\mathrm{Pi}$ & 3.179 & 3.183 & 3.129 & 3.134 & 3.168 & 3.1586 \\
\hline & elapsed time (seconds) & 64.253 & 64.205 & 64.221 & 64.237 & 64.126 & 64.2084 \\
\hline $10^{3}$ & value of $\mathrm{Pi}$ & 3.1428 & 3.1436 & 3.1457 & 3.1439 & 3.1412 & 3.14344 \\
\hline & elapsed time (seconds) & 64.233 & 64.233 & 64.233 & 64.233 & 64.233 & 64.233 \\
\hline $10^{4}$ & value of $\mathrm{Pi}$ & 3.1414 & 3.1409 & 3.1415 & 3.1425 & 3.1456 & 3.14238 \\
\hline & elapsed time (seconds) & 64.24 & 64.213 & 64.384 & 64.241 & 64.354 & 64.2864 \\
\hline $10^{5}$ & value of Pi & 3.1414 & 3.1417 & 3.1415 & 3.1412 & 3.146 & 3.14236 \\
\hline
\end{tabular}

As seen in Table 1 and Table 2, For Hama, the execution time is maintained at between 3.1 to 3.4 seconds. For Hadoop, the execution time is maintained at between 64.2 to 64.5 seconds. There is no apparent difference in total execution time between Hama 
and Hadoop algorithm. It can be inferred that the execution time of Hama is mainly consumed in the BSP function while the execution time of Hadoop is mainly consumed in the MapReduce functions when the number of iterations below $10^{5}$.

As seen from Fig.2, The computing efficiency of Hama is about 95 percent higher than that of Hadoop. This phenomenon is caused by the following reasons: Hama does not have a large amount of data read and write operations while Hadoop presences of a large number of $\mathrm{I} / \mathrm{O}$ operations.

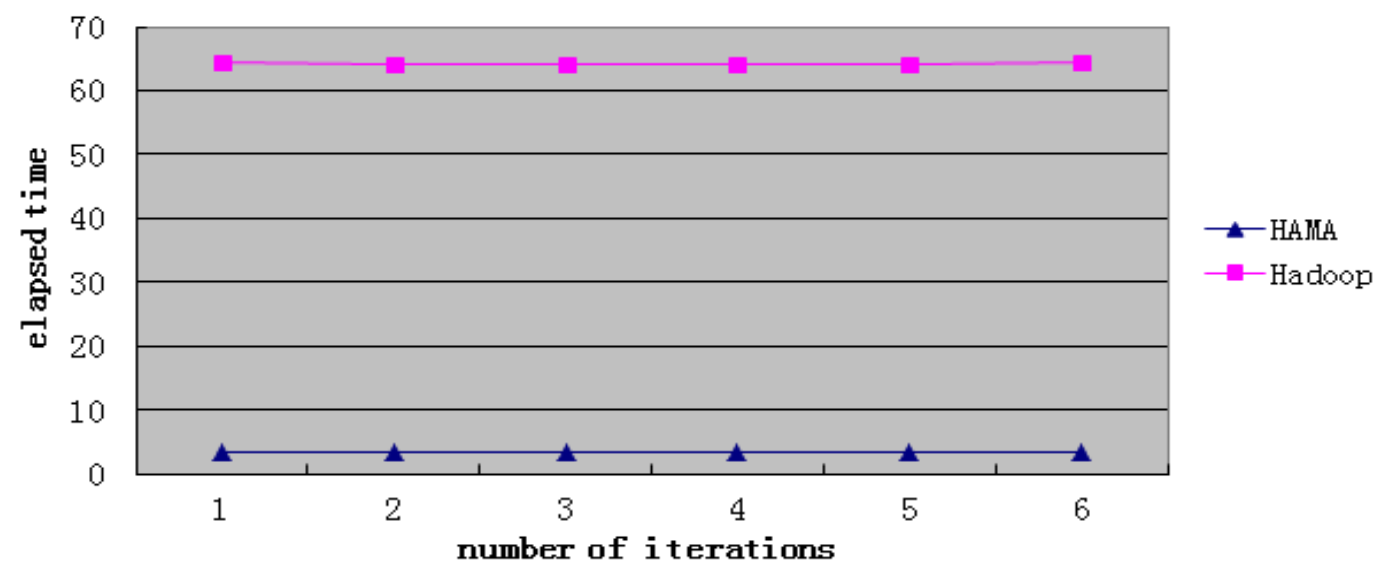

Figure 2. Advantage Interval

\subsection{Available Interval}

Table 3. Available Interval -Hama

\begin{tabular}{|c|c|c|c|c|c|c|c|}
\hline \multirow{2}{*}{ number of iterations } & \multicolumn{6}{|c|}{ Hama } & \multirow{2}{*}{$\begin{array}{l}\begin{array}{l}\text { mean } \\
\text { value }\end{array} \\
6.3662 \\
\end{array}$} \\
\hline & elapsed time (seconds) & 6.364 & 6.37 & 6.362 & 6.374 & 6.361 & \\
\hline $10^{6}$ & value of $\mathrm{Pi}$ & 3.1419 & 3.1413 & 3.1417 & 3.1417 & 3.142 & 3.14172 \\
\hline \multirow[t]{2}{*}{$2 \times 10^{6}$} & elapsed time (seconds) & 9.366 & 9.35 & 9.402 & 9.397 & 9.373 & 9.3776 \\
\hline & value of $\mathrm{Pi}$ & 3.1417 & 3.1417 & 3.1417 & 3.1416 & 3.1418 & 3.1417 \\
\hline \multirow[t]{2}{*}{$4 \times 10^{6}$} & elapsed time (seconds) & 18.307 & 18.384 & 18.322 & 18.308 & 18.36 & 18.3362 \\
\hline & value of $\mathrm{Pi}$ & 3.1416 & 3.1417 & 3.1417 & 3.1412 & 3.1413 & 3.1415 \\
\hline \multirow[t]{2}{*}{$6 \times 10^{6}$} & elapsed time (seconds) & 27.333 & 27.286 & 27.377 & 27.429 & 27.418 & 27.3686 \\
\hline & value of Pi & 3.1415 & 3.1416 & 3.1415 & 3.1415 & 3.1414 & 3.1415 \\
\hline \multirow[t]{2}{*}{$8 \times 10^{6}$} & elapsed time (seconds) & 36.33 & 37.091 & 36.357 & 36.435 & 36.658 & 36.5742 \\
\hline & value of $\mathrm{Pi}$ & 3.1416 & 3.1415 & 3.1414 & 3.1418 & 3.1414 & 3.14154 \\
\hline \multirow[t]{2}{*}{$10^{7}$} & elapsed time (seconds) & 45.376 & 44.992 & 45.651 & 45.241 & 42.576 & 44.7672 \\
\hline & value of $\mathrm{Pi}$ & 3.1416 & 3.1416 & 3.1417 & 3.1414 & 3.1415 & 3.14156 \\
\hline \multirow{2}{*}{$1.5 \times 10^{7}$} & elapsed time (seconds) & 66.346 & 66.417 & 67.753 & 66.195 & 66.628 & 66.6678 \\
\hline & value of $\mathrm{Pi}$ & 3.1415 & 3.1415 & 3.1411 & 3.1416 & 3.1419 & 3.14152 \\
\hline
\end{tabular}


Table 4. Available Interval - Hadoop

\begin{tabular}{|c|c|c|c|c|c|c|c|}
\hline \multirow{2}{*}{ number of iterations } & \multicolumn{6}{|l|}{ Hadoop } & \multirow{2}{*}{$\begin{array}{l}\text { mean value } \\
64.242\end{array}$} \\
\hline & elapsed time (seconds) & 64.242 & 64.254 & 64.252 & 64.256 & 64.352 & \\
\hline $10^{6}$ & value of $\mathrm{Pi}$ & 3.1415 & 3.1409 & 3.1412 & 3.1415 & 3.1416 & 3.14134 \\
\hline \multirow[t]{2}{*}{$2 \times 10^{6}$} & elapsed time (seconds) & 64.247 & 64.223 & 64.209 & 64.28 & 64.219 & 64.247 \\
\hline & value of $\mathrm{Pi}$ & 3.1415 & 3.1419 & 3.1414 & 3.1419 & 3.1415 & 3.14164 \\
\hline \multirow[t]{2}{*}{$4 \times 10^{6}$} & elapsed time (seconds) & 64.236 & 64.295 & 64.516 & 64.228 & 64.299 & 64.236 \\
\hline & value of Pi & 3.1413 & 3.1419 & 3.1414 & 3.1415 & 3.1415 & 3.14152 \\
\hline \multirow[t]{2}{*}{$6 \times 10^{6}$} & elapsed time (seconds) & 64.241 & 64.224 & 64.242 & 64.296 & 64.341 & 64.241 \\
\hline & value of $\mathrm{Pi}$ & 3.1415 & 3.1416 & 3.1418 & 3.1419 & 3.1414 & 3.14164 \\
\hline \multirow[t]{2}{*}{$8 \times 10^{6}$} & elapsed time (seconds) & 64.449 & 64.489 & 64.475 & 64.639 & 64.039 & 64.449 \\
\hline & value of $\mathrm{Pi}$ & 3.1415 & 3.1412 & 3.1419 & 3.1419 & 3.1415 & 3.1416 \\
\hline \multirow[t]{2}{*}{$10^{7}$} & elapsed time (seconds) & 64.377 & 64.392 & 64.757 & 64.354 & 64.257 & 64.4274 \\
\hline & value of $\mathrm{Pi}$ & 3.1416 & 3.1416 & 3.1416 & 3.1417 & 3.1416 & 3.14162 \\
\hline \multirow[t]{2}{*}{$1.5 \times 10^{7}$} & elapsed time (seconds) & 64.383 & 64.801 & 64.203 & 64.326 & 64.923 & 64.5272 \\
\hline & value of $\mathrm{Pi}$ & 3.1415 & 3.1416 & 3.1416 & 3.1417 & 3.1413 & 3.14154 \\
\hline
\end{tabular}

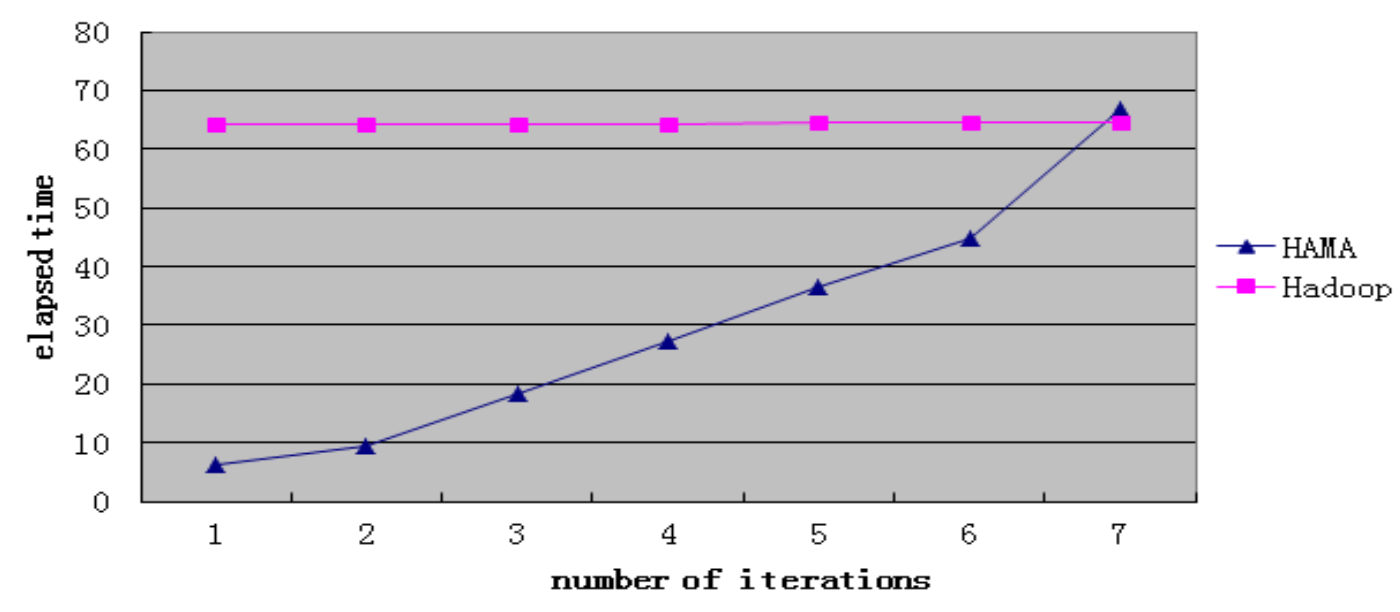

Figure 3. Available Interval

As seen in Table 3 and Table 4, For Hama, there is significant fluctuation in execution time. For Hadoop, the execution time to complete computing tasks is maintained at between 64.2 to 64.5 seconds.

As seen from Fig.3, the mainly execution time of Hama is iterative calculation while the execution time of BSP function is not increased significantly. The total execution time of Hadoop is mainly consumed in MapReduce functions when the number of iterations below $1.5 \times 10^{7}$. 
This is because Hama optimization operation focuses on BSP functions while there has no specialized optimization for the calculation process itself in Hama.

\subsection{Disadvantage Interval}

The experimental results are shown in Table 5 and Table 6, and graphed in Fig.4. Fig.4 shows significant performance degradation of the Hama implementation compared to Hadoop. This indicates that computing task itself consumes the main part of execution time when computation over a certain size.

The computing efficiency of Hadoop is about 35 percent higher than that of Hama. This phenomenon is caused by the following reasons: Intermediate results occupy a lot of hardware resources when the amount of calculation is increased. So, the hardware resources allocated to the MapReduce tasks and BSP tasks are substantially reduced. In the case, it leads to decline in operating efficiency for Hama and Hadoop.

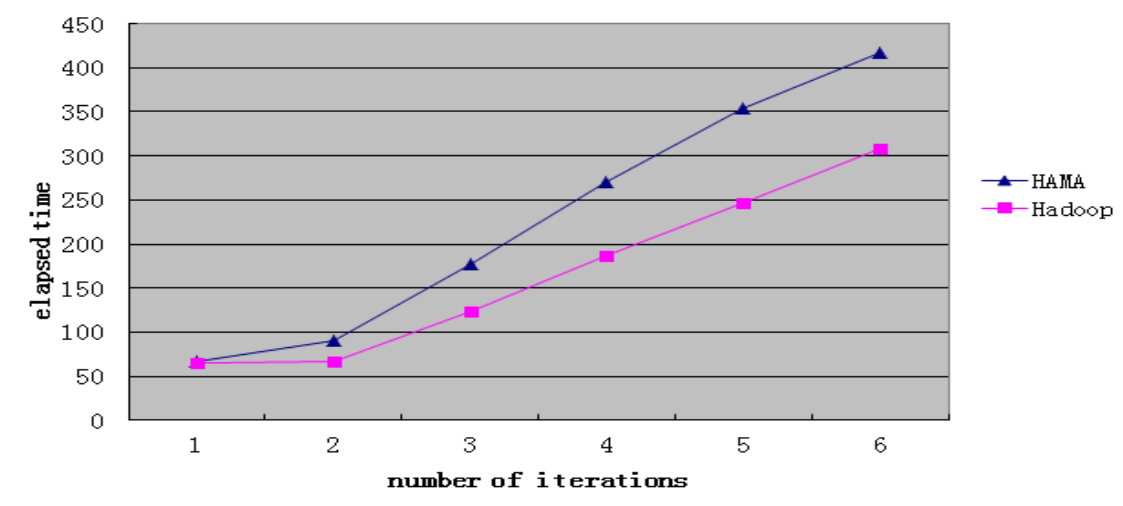

Figure 4. Disadvantage Interval

Table 5. Disadvantage Interval -Hama

\begin{tabular}{|c|c|c|c|c|c|c|c|}
\hline \multirow{2}{*}{ number of iterations } & \multicolumn{6}{|c|}{ Hama } & \multirow{2}{*}{$\begin{array}{l}\text { mean value } \\
66.6678\end{array}$} \\
\hline & elapsed time (seconds) & 66.346 & 66.417 & 67.753 & 66.195 & 66.628 & \\
\hline $1.5 \times 10^{7}$ & value of $\mathrm{Pi}$ & 3.1415 & 3.1415 & 3.1411 & 3.1416 & 3.1419 & 3.14152 \\
\hline \multirow[t]{2}{*}{$2 \times 10^{7}$} & elapsed time (seconds) & 89.435 & 89.761 & 89.348 & 91.825 & 89.419 & 89.9576 \\
\hline & value of $\mathrm{Pi}$ & 3.1417 & 3.1414 & 3.1412 & 3.1415 & 3.1415 & 3.14146 \\
\hline \multirow[t]{2}{*}{$4 \times 10^{7}$} & elapsed time (seconds) & 178.013 & 175.905 & 177.346 & 179.697 & 178.314 & 177.855 \\
\hline & value of $\mathrm{Pi}$ & 3.1415 & 3.1416 & 3.1415 & 3.1415 & 3.1413 & 3.14148 \\
\hline \multirow[t]{2}{*}{$6 \times 10^{7}$} & elapsed time (seconds) & 269.085 & 273.68 & 266.347 & 271.583 & 266.807 & 269.5004 \\
\hline & value of Pi & 3.1416 & 3.1414 & 3.1413 & 3.1418 & 3.1415 & 3.14152 \\
\hline \multirow[t]{2}{*}{$8 \times 10^{7}$} & elapsed time (seconds) & 356.368 & 348.584 & 355.018 & 360.139 & 352.634 & 354.5486 \\
\hline & value of $\mathrm{Pi}$ & 3.1415 & 3.1415 & 3.1415 & 3.1417 & 3.141 & 3.14144 \\
\hline \multirow[b]{2}{*}{$10^{8}$} & elapsed time (seconds) & 415.18 & 422.671 & 413.336 & 416.892 & 417.826 & 417.181 \\
\hline & value of $\mathrm{Pi}$ & 3.1415 & 3.1415 & 3.1415 & 3.1415 & 3.1418 & 3.14156 \\
\hline
\end{tabular}


Table 6. Disadvantage Interval -Hadoop

\begin{tabular}{|c|c|c|c|c|c|c|c|}
\hline \multirow{2}{*}{ number of iterations } & \multicolumn{6}{|c|}{ Hadoop } & \multirow{2}{*}{$\begin{array}{l}\text { mean value } \\
64.5272 \\
\end{array}$} \\
\hline & elapsed time (seconds) & 64.383 & 64.801 & 64.203 & 64.326 & 64.923 & \\
\hline $1.5 \times 10^{7}$ & value of $\mathrm{Pi}$ & 3.1415 & 3.1416 & 3.1416 & 3.1417 & 3.1413 & 3.14154 \\
\hline \multirow{2}{*}{$2 \times 10^{7}$} & $\begin{array}{l}\text { elapsed time } \\
\text { (seconds) }\end{array}$ & 67.419 & 67.094 & 67.849 & 67.419 & 67.443 & 67.4448 \\
\hline & value of $\mathrm{Pi}$ & 3.1415 & 3.1417 & 3.1416 & 3.1416 & 3.1415 & 3.14158 \\
\hline \multirow[t]{2}{*}{$4 \times 10^{7}$} & elapsed time (seconds) & 124.465 & 121.925 & 123.474 & 120.655 & 127.432 & 123.5902 \\
\hline & value of $\mathrm{Pi}$ & 3.1417 & 3.1415 & 3.1418 & 3.1413 & 3.1416 & 3.14158 \\
\hline \multirow[t]{2}{*}{$6 \times 10^{7}$} & elapsed time (seconds) & 184.548 & 187.842 & 182.788 & 185.606 & 189.517 & 186.0602 \\
\hline & value of $\mathrm{Pi}$ & 3.1416 & 3.1415 & 3.1416 & 3.1415 & 3.1412 & 3.14148 \\
\hline \multirow[t]{2}{*}{$8 \times 10^{7}$} & elapsed time (seconds) & 244.618 & 254.173 & 241.623 & 246.868 & 248.92 & 247.2404 \\
\hline & value of $\mathrm{Pi}$ & 3.1415 & 3.1415 & 3.1415 & 3.1414 & 3.1415 & 3.14148 \\
\hline \multirow[b]{2}{*}{$10^{8}$} & elapsed time (seconds) & 304.674 & 306.866 & 305.379 & 315.241 & 307.539 & 307.9398 \\
\hline & value of $\mathrm{Pi}$ & 3.1415 & 3.1415 & 3.1415 & 3.1416 & 3.1415 & 3.14152 \\
\hline
\end{tabular}

\section{Conclusions}

In this paper, we have evaluated the performance of Hama with Monte Carlo calculation of Pi. The results show that Hama shows higher computing performance than the Hadoop implementation when the number of iterations is less than a certain value $n$. The value of $\mathrm{n}$ depends on the experimental conditions. Otherwise, Hama has worse performance than Hadoop. We hope that the experimental results presented in this paper would be useful for the future development of Hama.

\section{Acknowledgments}

This work is supported by the National Natural Science Foundation of China (NSFC 61370060).

\section{References}

[1] B. Xu, C. Zhao, E. Hu, and B. Hu, "Job Scheduling Algorithm Based on Berger Model in Cloud environment", Advances in Engineering Software, vol. 42, no. 7, (2011),pp. 419-425.

[2] E.J. Yoon, J. Kim, S. Jin, J.-S. Kim, S. Maeng, "Hama: An Efficient Matrix Computation with the MapReduce Framework. 2010 IEEE Second International Conference on Cloud Computing Technology and Science", (2010), pp. 721-726.

[3] G. Malewicz, M. H. Austern, A.J.C Bik, J. C. Dehnert, I. Horn, N. Leiser, G. Czajkowski, "Pregel: a system for large-scale graph processing", ACM SIGMOD International Conference on Management of data, (2010), pp.135-146.

[4] "Apache Hadoop" en.wikipedia.org/wiki/Apache_Hadoop

[5] "Bulk Synchronous Parallel”, http://en.wikipedia.org/wiki/Bulk_synchronous_parallel

[6] P. Hunt, M. Konar, F. P. Junqueira, B. Reed, "ZooKeeper: Wait-free Coordination for Internet-scale Systems", USENIX Annual Technology Conference, (2010), pp.11-11.

[7] J. Dean and S. Ghemawat, "MapReduce: Simplified data processing on large clusters", Sixth Symposium on Operating System Design and Implementation, San Francisco, CA, December, 2004: 137-150.

[8] U. Kang, C. E. Tsourakakis, C. Faloutsos, "PEGASUS: Mining Peta-Scale Graphs", Knowledge and 
Information Systems, vol. 27, no. 2, (2011), pp.303-325.

[9] "JPregel", url: http://kowshik.github.com/JPregel/.

[10] "Apache Giraph", url: http : / /giraph .apache.org/

[11] E. Elnikety, T. Elsayed, H. E. Ramadan, "iHadoop: Asynchronous Iterations for MapReduce”, IEEE Third International Conference on Cloud Computing Technology and Science, Nov. 29 -Dec. 1 2011:81- 90.

[12] M. Isard, M. Budiu, Y. Yu, A. Birrell, D. Fetterly, "Dryad: Distributed Data-Parallel Programs from Sequential Building Blocks", The 2nd ACM SIGOPS/EuroSys European Conference on Computer Systems, (2007), pp. 59-72.

[13] "Twister", url: http://www.iterativeMapReduce.org/\#home.

[14] http://math.fullerton.edu/mathews/n2003/montecarloPimod.html.

[15] B. Xu, N. Wang, C. Li, "A cloud computing infrastructure on heterogeneous computing resources", Journal of Computers, (2011), vol. 6, no. 8, pp. 1789-1796.

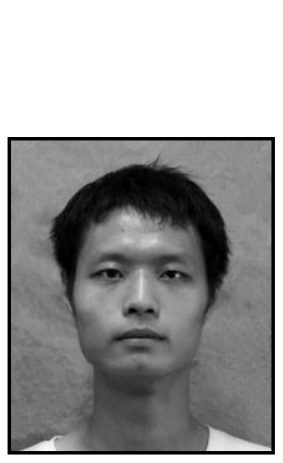

\section{Authors}

Shuo Li, he is an undergraduate student of Computer and Information Technology, Beijing Jiaotong University, China. He prefer to get a higher degree in the field of Cloud Computing, Big Data. He makes efforts to gain more attention of this field.

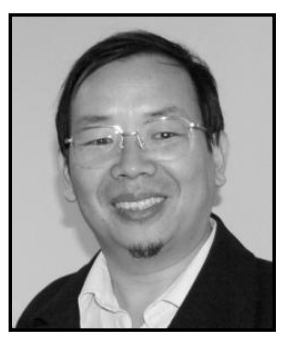

Baomin XU, Dr. Xu Baomin is an associate professor in School of Computer and Information Technology, Beijing Jiaotong University, China. His research interests include Large Scale Data Analysis Based-on Cloud Computing, and Complex Network (Link Prediction, Community Detection). Until now he has published more than 50 research papers in referred journals and proceedings. 